\title{
Drug-Drug Interactions between Direct Oral Anticoagulants and Hepatitis C Direct-Acting Antiviral Agents: Looking for Evidence Through a Systematic Review
}

\author{
Marta Bellesini ${ }^{1,2} \cdot$ Matteo Bianchin ${ }^{3} \cdot$ Chiara Corradi $^{4} \cdot$ Marco Paolo Donadini $^{1,2} \cdot$ Emanuel Raschi $^{3}$ (1) \\ Alessandro Squizzato ${ }^{1,2,4}$
}

Published online: 18 August 2020

(c) The Author(s) 2020

\begin{abstract}
Background Direct oral anticoagulants (DOACs), as substrates of cytochrome P450 (CYP) 3A4 and/or P-glycoprotein, are susceptible to drug-drug interactions (DDIs). Hepatitis C direct-acting antiviral agents (DAAs), via P-glycoprotein or CYP3A4 inhibition, may increase DOAC exposure with relevant bleeding risk. We performed a systematic review on DDIs between DOACs and DAAs.

Methods Two reviewers independently identified studies through electronic databases, until 7 July 2020, supplementing the search by reviewing conference abstracts and the ClinicalTrials.gov website.

Results Of 1386 identified references, four articles were finally included after applying the exclusion criteria. Three phase I clinical studies in healthy volunteers assessed interactions between dabigatran and glecaprevir/pibrentasvir, odalasvir/ simeprevir, or sofosbuvir/velpatasvir/voxilaprevir, showing an increase in the dabigatran area under the concentration-time curve (AUC) by $138 \%, 103 \%$, and $161 \%$, respectively.

Conclusions DOACs and DAAs are under-investigated for DDI risk. Real-world studies are needed to assess the clinical relevance of the pharmacokinetic interactions with dabigatran and describe the actual spectrum of possible DDIs between DAAs and other DOACs.
\end{abstract}

\section{Key Points}

This is the first systematic review assessing evidence about direct oral anticoagulants/direct-acting antiviral agents (DOACs/DAAs) drug-drug interactions (DDIs).

DAAs increase dabigatran concentration, while no studies were available for other DOACs.

Real-world studies are needed to evaluate clinical relevance of this interaction and to describe the actual spectrum of possible DDIs between DAAs and other DOACs.

Emanuel Raschi

emanuel.raschi@unibo.it

1 Department of Medicine and Surgery, Research Center on Thromboembolic Disorders and Antithrombotic Therapies, University of Insubria, Varese, Italy

2 Department of Medicine and Surgery, Research Center on Thromboembolic Disorders and Antithrombotic Therapies, University of Insubria, Como, Italy

\section{Introduction}

In recent years, the direct oral anticoagulants (DOACs) apixaban, dabigatran, edoxaban, and rivaroxaban have been progressively introduced worldwide. By virtue of their favorable pharmacological properties, including predictable pharmacokinetics [1], compared with vitamin $\mathrm{K}$ antagonists (VKAs), DOACs are recommended by major guidelines as first-choice anticoagulants for the treatment of venous thromboembolism (VTE) and stroke prevention in non-valvular atrial fibrillation (AF), as documented by current estimates on the incidence of new users [2-4].

Nevertheless, risk of bleeding remains a potentially serious complication and preventive strategies should be

Department of Medical and Surgical Sciences, Alma Mater Studiorum-University of Bologna, Via Irnerio 48, 40126 Bologna, Italy

4 Internal Medicine Unit, 'Sant' Anna' Hospital, San Fermo della Battaglia, Como, Italy 
targeted to real-world susceptible patients, largely underrepresented in clinical trials, such as extreme weights, severe kidney insufficiency, and concomitant administration of potentially interacting medications.

Among the various unsettled issues, the impact of polypharmacy and drug-drug interactions (DDIs) is perceived as a key research priority [5], also to disentangle the contribution of pharmacodynamic and pharmacokinetic mechanisms. Although DOACs have fewer DDIs than VKAs, significant interactions can still occur; DOACs are substrates of cytochrome P450 (CYP) 3A4 and/or P-glycoprotein $(\mathrm{P}-\mathrm{gp})$, making them susceptible to pharmacokinetic DDIs (see Table 1). In particular, coadministration of dabigatran and rifampicin, via P-gp induction, has been documented to lower dabigatran concentration by more than $60 \%$; coadministration of rivaroxaban with ketoconazole or ritonavir has instead been shown to raise rivaroxaban concentrations by more than $150 \%$, via CYP3A4 and P-gp/ $\mathrm{BCRP}$ (ABCG2) inhibition [6,7]. At present, there is no accepted threshold for a change in DOAC concentrations that defines clinical significance; however, the anticoagulant effect of DOACs is concentration-dependent, and significant changes in DOAC concentrations likely affects clinical outcomes [8].

While laboratory tests to assess the intensity of anticoagulation and the detection of DDIs are easily accessible for VKAs by monitoring the international normalized ratio, DOAC-specific tests and guidance for using them in special situations are now available with several limitations. In particular, there is actually no consensus on the therapeutic range of DOACs, even if information can be derived from phase II-III clinical trials regarding the 'ontherapy' range [9]. Based on data from pharmacokinetic studies, expert societies have proposed algorithms and thresholds for some clinical situations, which need to be validated in targeted prospective studies [9, 10].

In parallel with the increasing uptake of DOACs, several direct-acting antiviral agents (DAAs) for chronic hepatitis $\mathrm{C}$ virus (HCV) have been licensed in recent years; these agents have expanded the pharmacological armamentarium for treating HCV infection, with remarkable effectiveness and a good tolerability profile [11]. However, relevant treatment regimens contain at least two and up to five drugs, and carry potential metabolicor transport-mediated DDIs via P-gp or CYP3A4 inhibition (see Table 1) $[12,13]$. The potential for DDIs needs special consideration for $\mathrm{HCV}$-infected individuals with concomitant comorbidities, such as AF or VTE, requiring anticoagulant treatment.

Recent studies have suggested an increased risk of VTE among HCV-infected patients, coupled with an increased incidence of AF [14, 15], thus resulting in a likely rise in the number of DOAC prescriptions in the near future in patients with $\mathrm{HCV}$ infection. In addition, the use of DOACs in patients with liver disease is expected to increase in the next years as a result of the link between metabolic as well as viral liver disease and cardiovascular complications, and due to the concomitant increasing prevalence of nonalcoholic fatty liver disease and nonalcoholic steatohepatitis (NASH) [16]. In particular, on the one hand, HCV infection is often associated with both hepatic steatosis and with a specific HCV-associated dysmetabolic syndrome [17], while on the other hand, NASH currently represents the leading cause of chronic liver disease and is independently associated with an increased risk of cardiovascular diseases, namely cardiac rhythm disorders (mainly AF) and VTE [18-21]. Therefore, an increasing number of patients affected by chronic liver disease will be candidates for anticoagulant treatment with DOACs [16].

In patients with cirrhosis, the use of DOACs is controversial and challenging for a number of reasons, including (1) cirrhosis was an exclusion criteria of pivotal trials; (2) patients with cirrhosis are at risk of both bleeding and thrombotic complications; and (3) DOACs, especially rivaroxaban, have been associated with increased reporting of serious and early-onset liver injury [22, 23]. For these reasons, current guidelines and drug regulatory agencies (US FDA and European Medicines Agency) contraindicated all DOACs in patients with cirrhosis Child-Turcotte-Pugh (CTP) class $\mathrm{C}$, while variously allowing their use in patients with cirrhosis CTP class B and A [3, 24]. Emerging realworld data suggest that DOACs can effectively and safely be used in CTP A patients, and cautiously in CTP B cirrhotic patients, compared with VKAs [16], thus supporting the importance of an accurate evaluation of possible DDIs in $\mathrm{HCV}$ co-infected cirrhotic patients.

Because of these pharmacokinetic bases, the combination of DOACs and DAAs is not recommended, or is to be used with caution, as per major guidelines and online tools, albeit with little information on clinical relevance and relative proper management $[11,12]$.

We aimed to systematically assess the evidence regarding DDIs between DOACs and DAAs, and to provide adjunctive information on their clinical relevance, including management in clinical practice.

\section{Methods}

This systematic review was performed according to the Preferred Reporting Items for Systematic Reviews and Meta-Analyses (PRISMA) guidelines [25]. The protocol was designed a priori but was not submitted to public repositories. 
Table 1 Predicted pharmacokinetic drug interactions between the main hepatitis $\mathrm{C}$ direct-acting antiviral agents and direct oral anticoagulants

\begin{tabular}{|c|c|c|c|c|c|}
\hline & & \multicolumn{4}{|c|}{ DDIs with DOACs (PK prediction) } \\
\hline & & Dabigatran & Rivaroxaban & Apixaban & Edoxaban \\
\hline P-gp substrate & & Yes & Yes & No (minimal) & Yes \\
\hline CYP3A4 substrate & & No & Yes (moderate, $18 \%$ ) & Yes (moderate, $25 \%$ ) & No (minimal, $4 \%$ ) \\
\hline BCRP substrate & & No & Yes & Yes & No \\
\hline OATP1B1 substrate & & No & No & No & Yes \\
\hline $\begin{array}{l}\text { Hepatitis C direct-acting } \\
\text { antiviral agents }\end{array}$ & $\begin{array}{l}\text { PK pathway causing } \\
\text { DDIs }\end{array}$ & & & & \\
\hline Sofosbuvir & & No interaction expected & No interaction expected & No interaction expected & No interaction expected \\
\hline Sofosbuvir/ledipasvir & $\begin{array}{l}\text { Mild to moderate P-gp } \\
\text { inhibition (by ledi- } \\
\text { pasvir) } \\
\text { Weak inhibition of } \\
\text { OATPB1 (by ledi- } \\
\text { pasvir) }\end{array}$ & Potential interaction & Potential interaction & Potential interaction & Potential interaction \\
\hline Sofosbuvir/velpatasvir & $\begin{array}{l}\text { Mild P-gp inhibition (by } \\
\text { velpatasvir) } \\
\text { Inhibition of BCRP (by } \\
\text { velpatasvir) }\end{array}$ & Potential interaction & Potential interaction & Potential interaction & Potential interaction \\
\hline $\begin{array}{l}\text { Ombitasvir/paritaprevir/ } \\
\text { ritonavir + dasabuvir }\end{array}$ & $\begin{array}{l}\text { P-gp inhibition } \\
\text { Inhibition of CYP3A4 } \\
\text { (by paritaprevir and } \\
\text { ritonavir) } \\
\text { Inhibition of BCRP (by } \\
\text { ritonavir and dasa- } \\
\text { buvir) } \\
\text { Inhibition of OATP1B1 } \\
\text { (by paritaprevir) }\end{array}$ & Potential interaction & $\begin{array}{l}\text { Avoid coadministration } \\
\text { (not recommended) }\end{array}$ & $\begin{array}{l}\text { Avoid coadministration } \\
\text { (not recommended) }\end{array}$ & Potential interaction \\
\hline Grazoprevir/elbasvir & $\begin{array}{l}\text { Weak inhibition of } \\
\text { CYP3A4 (by grazo- } \\
\text { previr) } \\
\text { Mild P-gp inhibition (by } \\
\text { elbasvir) } \\
\text { Inhibition of BCRP (by } \\
\text { elbasvir/grazoprevir) }\end{array}$ & Potential interaction & Potential interaction & Potential interaction & Potential interaction \\
\hline $\begin{array}{l}\text { Sofosbuvir/velpatasvir/ } \\
\text { voxilaprevir }\end{array}$ & $\begin{array}{l}\text { Mild P-gp inhibition } \\
\text { (by velpatasvir and } \\
\text { voxilaprevir) } \\
\text { Inhibition of BCRP (by } \\
\text { velpatasvir and voxil- } \\
\text { aprevir) } \\
\text { Inhibition of OATP1B1 } \\
\text { (by velpatasvir and } \\
\text { voxilaprevir) }\end{array}$ & $\begin{array}{l}\text { Avoid coadministration } \\
\text { (not recommended) }\end{array}$ & Potential interaction & Potential interaction & $\begin{array}{l}\text { Avoid coadministration } \\
\text { (not recommended) }\end{array}$ \\
\hline Glecaprevir/pibrentasvir & $\begin{array}{l}\text { Weak inhibition of } \\
\text { CYP3A4 } \\
\text { Strong inhibition of P-gp } \\
\text { Inhibition of BCRP }\end{array}$ & $\begin{array}{l}\text { Avoid coadministration } \\
\text { (not recommended) }\end{array}$ & Potential interaction & Potential interaction & Potential interaction \\
\hline Simeprevir & $\begin{array}{l}\text { Mild-moderate P-gp } \\
\text { inhibition } \\
\text { Inhibition of CYP3A4 } \\
\text { Inhibition of OATPB1 }\end{array}$ & Potential interaction & Potential interaction & Potential interaction & Potential interaction \\
\hline
\end{tabular}

Based on https://www.hep-druginteractions.org (University of Liverpool) [12] and Talavera Pons et al. [13]

CYP cytochrome P450, P-gp P-glycoprotein, BCRP breast cancer resistance protein, $P K$ pharmacokinetic, DOACs direct oral anticoagulants, $D D I s$ drug-drug interactions, $O A T P$ organic anion-transporting polypeptide

We searched studies that assessed DDIs between DOACs and DAAs, using the MEDLINE (1966 to 7 July 2020) and EMBASE (1980 to 7 July 2020) electronic databases. The search strategy was developed without any language restriction using https://www.embase.com: 'antiinfective agent' AND 'hepatitis c' AND 'anticoagulant agent'.

We supplemented our search by handsearching unpublished literature, including the ClinicalTrials.gov website 
and congress abstracts of the International Society on Thrombosis and Haemostasis (ISTH), European Association for the Study of the Liver (EASL), International Society of Pharmacovigilance (ISoP), International Society for Pharmacoepidemiology (ISPE), and the European Association for Clinical Pharmacology and Therapeutics (EACPT).

Two investigators (MB and $\mathrm{MB}$ ) independently performed study selection. According to prespecified selection criteria, articles were included if (1) the study evaluated potential interaction between DOACs and DAAs; and (2) outcome measures were reported. Disagreements were resolved through discussion with a third reviewer (AS).

Two reviewers independently extracted data on study and population characteristics, type of intervention and outcome measures.

We established a priori to use the Cochrane ROBINS-I and RoB 2 tools for quality assessment but they were not applicable due to the type of included studies [26, 27].

\section{Results}

We identified 1385 references through a systematic database search. After applying inclusion criteria, three studies (Kosloski et al. [28], Boyle et al. [29], and OuwerkerkMahadevan et al. [30]) were eligible for inclusion. One additional study was identified through handsearching of EASL conference abstracts (Garrison et al. [31]). Of four articles included in this systematic review, only one was available in full text [28]. A PRISMA flow diagram detailing the screening process is shown in Fig. 1.
Of the included studies, three were phase I studies conducted in healthy volunteers, each assessing interactions between dabigatran and glecaprevir/pibrentasvir, odalasvir/ simeprevir, or sofosbuvir/velpatasvir/voxilaprevir [28, 30, 31]. Characteristics of the included studies and outcome measures are reported in Table 2.

Increased dabigatran concentrations during coadministration with DAAs was found, with the area under the concentration-time curve (AUC) increasing by $138 \%, 103 \%$, and $161 \%$, respectively.

One study was a retrospective observational study, with the aim of reviewing management strategies adopted by clinicians to handle DDIs. The study was conducted in a population of 54 patients taking DOACs and DAAs, over a 1 -year period [29]. Clinical monitoring was the most common strategy (no serious bleeding events were reported).

\section{Discussion}

To the best of our knowledge, this is the first systematic review aimed at evaluating DDIs between DOACs and DAAs. The following key findings can be identified. First, there are no postmarketing real-world data specifically investigating the clinical impact of DOAC-DAA combinations; second, notwithstanding the systematic search, including several gray areas of the literature, only a few studies have assessed these DDIs from a pharmacokinetic perspective only; third, for dabigatran only, dedicated drug interaction studies were retrieved without information on rivaroxaban, abixaban or edoxaban (the most frequently used DOACs in current clinical practice).
Fig. 1 PRISMA flow diagram of the study selection process (included and excluded studies). DOACs direct oral anticoagulants, DAAs direct-acting antiviral agents for chronic hepatitis C, DDIs drug-drug interactions, $V K A$ vitamin $\mathrm{K}$ antagonist
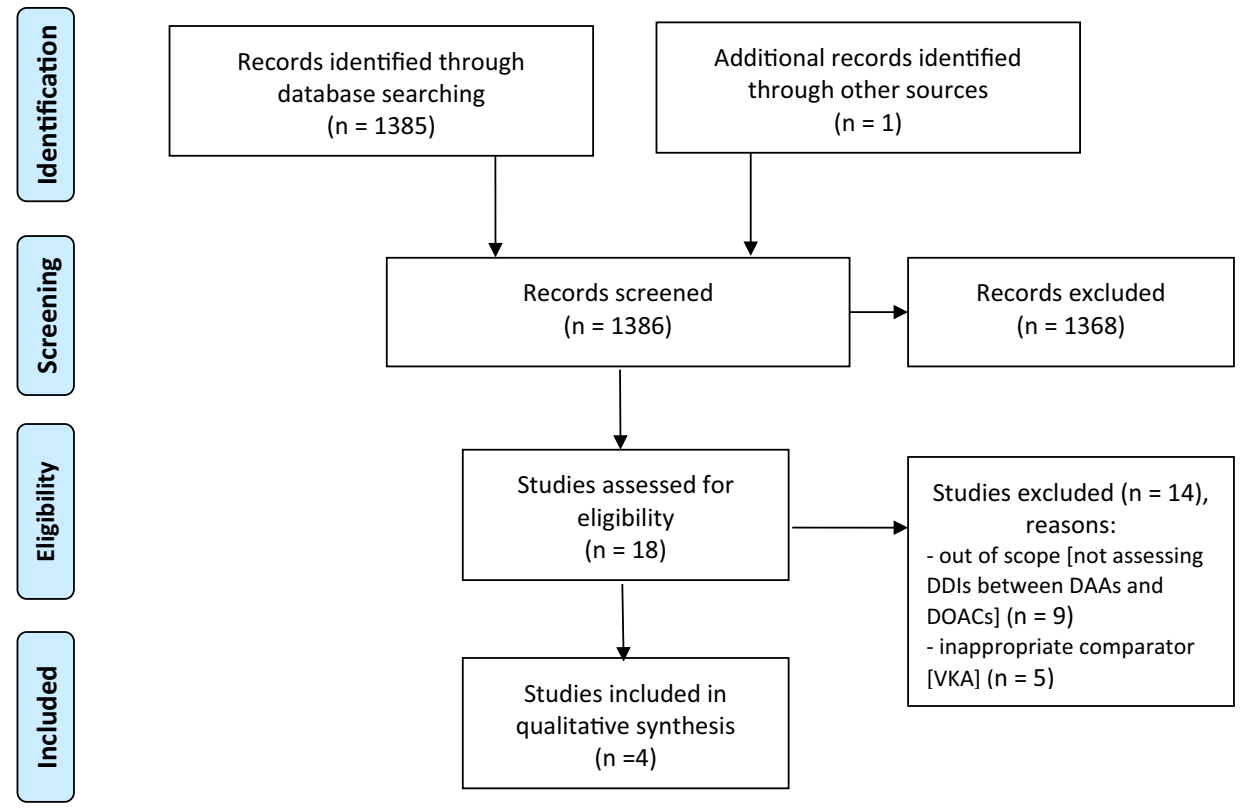


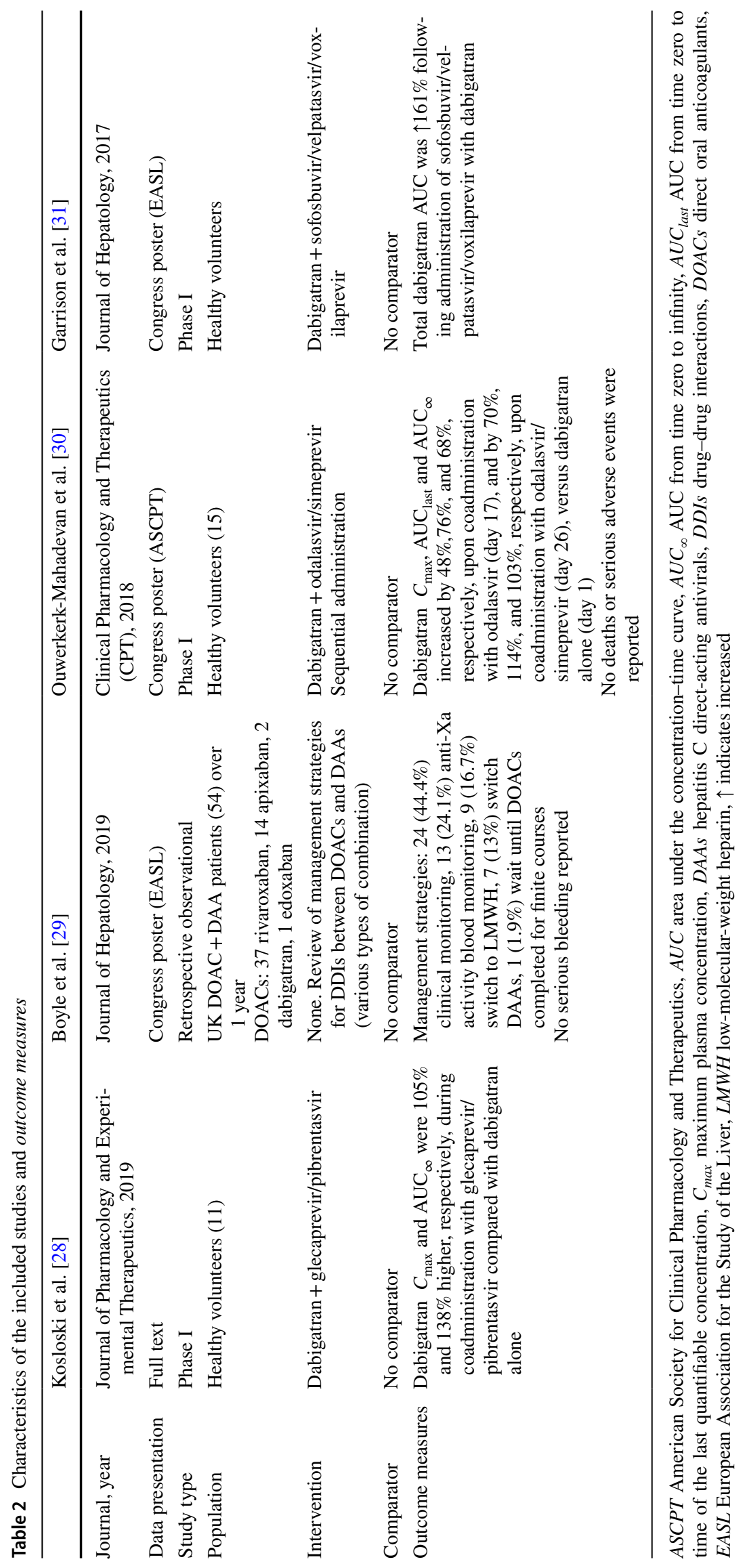


Overall, the paucity of data urgently calls for dedicated real-world studies to verify the actual clinical relevance of the pharmacokinetic interactions with dabigatran, and investigate the real risk in users of anti-factor Xa drugs, both in terms of bleeding risk and occurrence of liver injury. In fact, both rivaroxaban and apixaban are P-gp and BCRP substrates, whereas the active substrate of edoxaban is also a substrate of OATP1B1, thus making clinically important drug interactions highly likely.

The new oral hepatitis $\mathrm{C}$ drugs are examples where interpretation of a potential interaction may be particularly complex due to multiple drugs with different pharmacokinetic profiles concomitantly administered [32]. For instance, ledipasvir and sofosbuvir are P-gp substrates in vitro (and ledipasvir is also a P-gp inhibitor), whereas paritaprevir, ritonavir and dasabuvir all inhibit P-gp in vitro. Our data (even a twofold increased AUC of dabigatran) strongly suggest the clinical importance of this pharmacokinetic interaction. Apart from additional stringent clinical monitoring of susceptible patients (e.g. elderly with organ impairment receiving polypharmacy), outdistanced administration of the object and precipitant drug could be a viable option to minimize the likelihood of a pharmacokinetic interaction between dabigatran and DAAs, as previously demonstrated with verapamil (a P-gp and CYP3A4 inhibitor); administration of dabigatran etexilate (the prodrug) $2 \mathrm{~h}$ before verapamil did not significantly increase exposure to dabigatran etexilate $(<20 \%$ increase in AUC) [33].

Notably, there are key differences in the pharmacokinetic profiles among DOACs; apart from P-gp interactions (with dabigatran, as a prodrug, more likely to be affected), the extent of hepatic metabolism varies substantially, with rivaroxaban and apixaban undergoing nonnegligible (65-75\%) liver pathways, mediated for $25 \%$ by CYP3A4/5 and CYP2J2 [34]. Therefore, real-world studies are essential to verify the actual bleeding risk in users of DOACs and DAAs, especially considering that different DAAs possess additional properties on CYP3A4 (inhibitors or substrates), and the potential mismatch between pharmacokinetic data and observed associations in population-based studies [32, 35]. For dabigatran (in patients aged $>85$ years), recent cohort studies on rivaroxaban and edoxaban found increased bleeding occurrence in patients exposed to P-gp inhibitors and moderate CYP3A4 inhibitors, as also expected from clinical trials, where frequencies of bleeding events and mortality, but not stroke and systemic embolism, were increased with the increasing number of concomitant drugs [36-38].

Moreover, as shown in our review, DDIs between dabigatran and the DAAs tested resulted in a change of dabigatran concentration $>25 \%$; this cut-off was used in a previous review assessing DDIs of DOACs as a clinically significant change for maximum concentration $\left(C_{\max }\right)$ and
AUC, in line with FDA guidance [39]. The actual relationship between these pharmacokinetic bases and clinical outcomes is still debated.

We noted partial disagreement between international guidelines; the EASL guidelines contraindicated some combinations of DAAs/DOACs, whereas there is no specific mention in the 2018 European Heart Rhythm Association (EHRA) guidelines, and only coadministration of an HIV protease inhibitor (e.g. ritonavir) with DOACs is not recommended $[3,11]$. Our findings may support an evidencebased update of future guidelines, in particular regarding coadministration of dabigatran with glecaprevir/pibrentasvir, odalasvir/simeprevir, or sofosbuvir/velpatasvir/voxilaprevir. However, with regard to DDIs between DOACs and antiretroviral HIV treatment (sharing similar predicted pharmacokinetic DDIs with DAAs), in a recent small series of 14 HIV-infected patients concurrently treated with a variety of an antiretroviral and dabigatran for AF, dabigatran levels remained within the population expected range [40]. This further highlights the need for real-world targeted studies to assess the clinical relevance of the pharmacokinetic interaction that emerged in our review between dabigatran and HCV DAAs.

The strengths of our study include its systematic approach, including the implementation of our search with gray literature, through handsearching of conference abstracts. However, limitations of this systematic review should be acknowledged, which are mainly related to study design and full-text availability.

Apart from the very limited number of available studies, most studies were pharmacokinetic phase I trials, conducted in a small number of healthy volunteers. However, we believe this limitation should further raise awareness of clinicians on the risk of bleeding in subjects with chronic $\mathrm{HCV}$ and concomitant diseases, such as AF or VTE, taking several medications.

Due to the study design, a comparator was not tested. Therefore, no conclusions can be drawn as to whether the use of other anticoagulant treatments (such as heparin or VKAs), instead of DOACs, would bring benefit in this context. Although no major complications or major bleeding were reported in the studies included, also considering the small number of subjects enrolled, no firm conclusions can be made on the clinical relevance of these results due to the lack of consensus on clinically relevant changes in DOAC concentrations correlating with actual clinical outcomes.

Lastly, little information was available in the literature on how to manage potential DDIs between DOACs and DAAs in clinical practice; the only study available was a small observational study showing heterogeneity in the approaches used by clinicians to handle DDIs between DOACs and DAAs [41]. Therefore, our results further highlight the 
lack of data available and the urgent conduction of targeted studies.

\section{Conclusions}

The paucity of data documented by this systematic review underline the need to assess the actual clinical impact of anticipated pharmacokinetic interactions between DOACs and DAAs through real-world studies, thus supporting clinicians in safe prescribing.

Acknowledgements Open access funding provided by Alma Mater Studiorum - Universitá di Bologna within the CRUI-CARE Agreement. This systematic review was presented as an e-poster at the ISTH 2020 Virtual Congress (12-14 July 2020).

Author Contributions MB was responsible for the conceptualization and study design, data extraction, data presentation, data interpretation, and manuscript drafting and editing. $\mathrm{MB}$ was responsible for the data extraction, data presentation, and data interpretation. $\mathrm{CC}$ was responsible for the data interpretation. MPD was responsible for the conceptualization and study design, and data interpretation. ER was responsible for the conceptualization and study design, data interpretation, and manuscript drafting and editing. AS supervised the project and is guarantor for the study; he was responsible for the conceptualization and study design, data interpretation, and manuscript editing. All authors provided substantial contributions to data interpretation and discussion. They critically revised the content and approved the final version of the manuscript.

\section{Declarations}

Funding This work was supported by institutional research funds.

Conflict of interest Marta Bellesini, Matteo Bianchin, Chiara Corradi, and Marco Paolo Donadini declare they have no conflicts of interest. Emanuel Raschi reports personal fees from Novartis, outside the submitted work. Alessandro Squizzato received fees for lectures and/or advisory board meetings from Daiichi Sankyo, Pfizer, Bristol Myers Squibb, Bayer, and Boehringer Ingelheim.

Ethics approval Not applicable.

Consent to participate Not applicable.

Consent for publication Not applicable.

Availability of data and material Data sharing is not applicable to this article as no new data were created or analyzed in this study.

Code availability Not applicable.

Open Access This article is licensed under a Creative Commons Attribution-NonCommercial 4.0 International License, which permits any non-commercial use, sharing, adaptation, distribution and reproduction in any medium or format, as long as you give appropriate credit to the original author(s) and the source, provide a link to the Creative Commons licence, and indicate if changes were made. The images or other third party material in this article are included in the article's Creative Commons licence, unless indicated otherwise in a credit line to the material. If material is not included in the article's Creative Commons licence and your intended use is not permitted by statutory regulation or exceeds the permitted use, you will need to obtain permission directly from the copyright holder. To view a copy of this licence, visit http://creativecommons.org/licenses/by-nc/4.0/.

\section{References}

1. Raschi E, Bianchin M, Ageno W, De Ponti R, De Ponti F. Riskbenefit profile of direct-acting oral anticoagulants in established therapeutic indications: an overview of systematic reviews and observational studies. Drug Saf. 2016;39:1175-87.

2. Kearon C, Akl EA, Ornelas J, et al. Antithrombotic therapy for VTE disease: CHEST guideline and expert panel report. Chest. 2016;149:315-52.

3. Steffel J, Verhamme P, Potpara TS, et al. The 2018 European Heart Rhythm Association practical guide on the use of nonvitamin $\mathrm{K}$ antagonist oral anticoagulants in patients with atrial fibrillation. Eur Heart J. 2018;39:1330-933.

4. Ibáñez L, Sabaté M, Vidal X, et al. Incidence of direct oral anticoagulant use in patients with nonvalvular atrial fibrillation and characteristics of users in 6 European countries (2008-2015): a cross-national drug utilization study. Br J Clin Pharmacol. 2019;85:2524-39.

5. Raschi E, Bianchin M, Gatti M, Squizzato A, De Ponti F. Comparative effectiveness and safety of direct oral anticoagulants: overview of systematic reviews. Drug Saf. 2019;42:1409-22.

6. Härtter S, Koenen-Bergmann M, Sharma A, Nehmiz G, Lemke U, Timmer W, et al. Decrease in the oral bioavailability of dabigatran etexilate after co-medication with rifampicin. Br J Clin Pharmacol. 2012;74:490-500.

7. Mueck W, Kubitza D, Becka M. Co-administration of rivaroxaban with drugs that share its elimination pathways: pharmacokinetic effects in healthy subjects. Br J Clin Pharmacol. 2013;76:455-66.

8. Testa S, Legnani C, Antonucci E, et al. Drug levels and bleeding complications in atrial fibrillation patients treated with direct oral anticoagulants. J Thromb Haemost. 2019;17:1064-72.

9. Douxfils J, Ageno W, Samama C-M, Lessire S, ten Cate H, Verhamme $\mathrm{P}$, et al. Laboratory testing in patients treated with direct oral anticoagulants: a practical guide for clinicians. J Thromb Haemost. 2018;16:209-19.

10. Tripodi A, Ageno W, Ciaccio M, et al. Position paper on laboratory testing for patients on direct oral anticoagulants. A consensus document from the SISET, FCSA, SIBioC and SIPMeL. Blood Transfus. 2018;16:462-70.

11. European Association for the Study of the Liver. EASL recommendations on treatment of hepatitis C 2018. J Hepatol. 2018;69:461-511.

12. University of Liverpool, HEP drug interactions. Liverpool HEP interactions. https://www.hep-druginteractions.org/. Accessed 22 July 2020.

13. Talavera Pons S, Boyer A, Lamblin G, Chennell P, Châtenet $\mathrm{F}$, Nicolas $\mathrm{C}$, et al. Managing drug-drug interactions with new direct-acting antiviral agents in chronic hepatitis C. Br J Clin Pharmacol. 2017;83:269-93.

14. Wijarnpreecha K, Thongprayoon C, Panjawatanan P, Ungprasert $\mathrm{P}$. Hepatitis $\mathrm{C}$ virus infection and risk of venous thromboembolism: a systematic review and meta-analysis. Ann Hepatol. 2017;16:514-20.

15. Yang Y-H, Chiang H-J, Yip H-K, Chen K-J, Chiang JY, Lee MS, et al. Risk of new-onset atrial fibrillation among Asian chronic hepatitis $\mathrm{C}$ virus carriers: a nationwide population-based cohort study. J Am Heart Assoc. 2019;8:e012914. 
16. Ballestri S, Capitelli M, Fontana MC, Arioli D, Romagnoli E, Graziosi C, et al. Direct oral anticoagulants in patients with liver disease in the era of non-alcoholic fatty liver disease global epidemic: a narrative review. Adv Ther. 2020;37:1910-32.

17. Ballestri S, Nascimbeni F, Romagnoli D, Baldelli E, Targher G, Lonardo A. Type 2 diabetes in non-alcoholic fatty liver disease and hepatitis $\mathrm{C}$ virus infection-liver: the "musketeer" in the spotlight. Int J Mol Sci. 2016;17:355.

18. Spinosa M, Stine JG. Nonalcoholic fatty liver disease-evidence for a thrombophilic state? Curr Pharm Des. 2020;26:1036-44.

19. Ballestri S, Lonardo A, Bonapace S, Byrne CD, Loria P, Targher G. Risk of cardiovascular, cardiac and arrhythmic complications in patients with non-alcoholic fatty liver disease. World J Gastroenterol. 2014;20:1724-45.

20. Anstee QM, Mantovani A, Tilg H, Targher G. Risk of cardiomyopathy and cardiac arrhythmias in patients with nonalcoholic fatty liver disease. Nat Rev Gastroenterol Hepatol. 2018;15:425-39.

21. Di Minno MND, Tufano A, Rusolillo A, Di Minno G, Tarantino G. High prevalence of nonalcoholic fatty liver in patients with idiopathic venous thromboembolism. World J Gastroenterol. 2010;16:6119-222.

22. Raschi E, Bianchin M, De Ponti R, De Ponti F, Ageno W. Emerging therapeutic uses of direct-acting oral anticoagulants: an evidence-based perspective. Pharmacol Res. 2017;120:206-18.

23. Raschi E, Poluzzi E, Koci A, Salvo F, Pariente A, Biselli M, et al. Liver injury with novel oral anticoagulants: assessing post-marketing reports in the US Food and Drug Administration adverse event reporting system. Br J Clin Pharmacol. 2015;80:285-93.

24. Qamar A, Vaduganathan M, Greenberger NJ, Giugliano RP. Oral anticoagulation in patients with liver disease. J Am Coll Cardiol. 2018;71:2162-75.

25. Shamseer L, Moher D, Clarke M, Ghersi D, Liberati A, Petticrew M, PRISMA-P Group, et al. Preferred reporting items for systematic review and meta-analysis protocols (PRISMA-P) 2015: elaboration and explanation. BMJ. 2015;350:g7647.

26. Sterne JA, Hernán MA, Reeves BC, et al. ROBINS-I: a tool for assessing risk of bias in non-randomised studies of interventions. BMJ. 2016;355:i4919.

27. Sterne JAC, Savović J, Page MJ, et al. RoB 2: a revised tool for assessing risk of bias in randomised trials. BMJ. 2019;366:14898.

28. Kosloski MP, Bow DAJ, Kikuchi R, Wang H, Kim EJ, Marsh K, et al. Translation of in vitro transport inhibition studies to clinical drug-drug interactions for glecaprevir and pibrentasvir. J Pharmacol Exp Ther. 2019;370:278-87.

29. Boyle A, Davidson K, Cassidy C, et al. THU-130-management strategies for drug drug interactions between direct oral anticoagulants and hepatitis $\mathrm{C}$ directly acting agents: a multicentre review. J Hepatol. 2019;70:e216-e217217.
30. Ouwerkerk-Mahadevan S, Gamil M, Van Hemelryck S, Hillewaert $\mathrm{V}$, Biermer M. Pharmacokinetic interaction between the P-glycoprotein substrate dabigatran etexilate and HCV direct-acting antiviral agents, odalasvir and simeprevir. Clin Pharmacol Ther. 2018;103:S84.

31. Garrison KL, Kirby B, Stamm LM, Ma G, Vu A, Ling J. Drugdrug interaction profile of sofosbuvir/velpatasvir/voxilaprevir fixed-dose combination. J Hepatol. 2017;66:S492-S493493.

32. Lund M, Petersen TS, Dalhoff KP. Clinical Implications of P-glycoprotein modulation in drug-drug interactions. Drugs. 2017;77:859-83.

33. Härtter S, Sennewald R, Nehmiz G, Reilly P. Oral bioavailability of dabigatran etexilate (Pradaxa $\left({ }^{\circledR}\right)$ ) after co-medication with verapamil in healthy subjects. Br J Clin Pharmacol. 2013;75:1053-62.

34. Gelosa P, Castiglioni L, Tenconi M, Baldessin L, Racagni G, Corsini A. Pharmacokinetic drug interactions of the non-vitamin $\mathrm{K}$ antagonist oral anticoagulants (NOACs). Pharmacol Res. 2018;135:60-79.

35. Chang S-H, Chou I-J, Yeh Y-H, Chiou M-J, Wen M-S, Kuo $\mathrm{C}-\mathrm{T}$, et al. Association Between use of non-vitamin $\mathrm{K}$ oral anticoagulants with and without concurrent medications and risk of major bleeding in nonvalvular atrial fibrillation. JAMA. 2017;318:1250-9.

36. Bernier M, Lancrerot S-L, Rocher F, Van-Obberghen EK, Olivier $\mathrm{P}$, Lavrut $\mathrm{T}$, et al. Major bleeding events in octagenarians associated with drug interactions between dabigatran and P-gp inhibitors. J Geriatr Cardiol. 2019;16:806-11.

37. Hanigan S, Das J, Pogue K, Barnes GD, Dorsch MP. The real world use of combined P-glycoprotein and moderate CYP3A4 inhibitors with rivaroxaban or apixaban increases bleeding. J Thromb Thrombolysis. 2020;49:636-43.

38. Harskamp RE, Teichert M, Lucassen WAM, van Weert HCPM, Lopes RD. Impact of polypharmacy and P-glycoprotein- and CYP3A4-modulating drugs on safety and efficacy of oral anticoagulation therapy in patients with atrial fibrillation. Cardiovasc Drugs Ther. 2019;33:615-23.

39. Herink MC, Zhuo YF, Williams CD, DeLoughery TG. Clinical management of pharmacokinetic drug interactions with direct oral anticoagulants (DOACs). Drugs. 2019;79:1625-34.

40. Perram J, O'Dwyer E, Holloway C. Use of dabigatran with antiretrovirals. HIV Med. 2019;20:344-6.

41. Testa S, Ageno W, Antonucci E, et al. Management of major bleeding and outcomes in patients treated with direct oral anticoagulants: results from the START-Event registry. Intern Emerg Med. 2018;13:1051-8. 